CIENCIA Y SOCIEDAD

Volumen XXVIII, Número 2

Abril-Junio 2003

\title{
LA BANCA DOMINICANA Y LOS MÁRGENES DE INTERMEDIACIÓN
}

\begin{abstract}
Alberto Veloz*
\section{RESUMEN}

La banca local ha registrado cambios en los noventa, que han implicado fusiones y adquisiciones. Estos cambios alteran las participaciones en el mercado; sin embargo, la concentración de la mayor parte de los depósitos del público en un pequeño número de bancos se mantiene con la existencia de poder de mercado. El poder de mercado se asocia con elevados márgenes de intermediación; por tanto, elevadas tasas activas. La concentración en la banca local es superior a niveles observados en otros países latinos y a los registrados en las economías pequeñas del Caribe anglófono. La competencia monopolística que caracteriza la banca local contribuye a mantener tasas activas elevadas. El desarrollo de la bolsa de valores y la entrada al mercado de competidores con tamaño apreciable, contribuiría a la reducción de las tasas activas.
\end{abstract}

\section{PALABRAS CLAVES}

Poder de mercado, margen de intermediación, estimación VAR.

* El autor es profesor de Intec. Se agradece a lngrid Isidor su colaboración con los datos y las estimaciones VAR. 


\section{PRÓlOGO}

En un artículo anterior se sometió a pruebas econométricas la influencia que los intermediarios financieros pueden tener sobre las tasas de interés. Específicamente, se observó como la concentración de la mayor parte de los depósitos, en unos pocos intermediarios, está estrechamente asociado con elevados márgenes de intermediación, por ende, elevadas tasas de interés.

Las pruebas se realizaron con datos de las Asociaciones de Ahorro y Préstamos. Este grupo de intermediarios financieros pasa actualmente por un período de transición. De ser un grupo especializado en el financiamiento para la vivienda, el recién aprobado código monetario establece un plazo de un año para la elaboración de una ley particular para éstas. Diferentes resultados pueden esperarse, pero es inminente de que las Asociaciones de mayor tamaño ampliarán la gama de productos que ofrecen incluyendo algunos, que hasta la fecha ofrecen sólo los bancos comerciales.

El mercado financiero se ha visto sometido a cambios donde las adquisiciones y fusiones bancarias se han registrado con cierta frecuencia. En el año 1994, los reportes de La Superintendencia de Bancos mostraban datos para un total de 16 bancos. Al cierre del 2000, sólo habían 13. El número de Asociaciones es de 18. Sin embargo, los bancos administran un $78.6 \%$ de los activos del sistema financiero, de acuerdo a las cifras de la Superintendencia al 31 de diciembre del 2002. Las Asociaciones administran un $16 \%$. El segmento del mercado financiero que corresponde a las Asociaciones es más estable en cuanto al número de instituciones.

Factores tales como: elevadas tasas de interés, frecuentes restricciones sobre la liquidez del sector privado, depreciación del tipo de cambio y la necesidad de un mayor desarro- 
llo de los mecanismos de información de crédito, también presionan hacia arriba los costos de intermediación financiera. El poder de mercado puede explicar la transferencia de los aumentos en los costos de intermediación, tanto financieros como no financieros, a los usuarios del servicio; vía altas tasas activas.

Elevados intereses y márgenes de intermediación (MI), también se observan al el sector de la banca comercial. Es innegable que tasas de interés elevadas, por encima de las tasas prevalecientes en los mercados de los países con los que tenemos intercambio comercial, nos hace menos competitivos. Por otra parte, los elevados márgenes de intermediación penalizan el ahorro y limitan proyectos de inversión, con plazos de recuperación de mas de dos o tres años. Muchos de los proyectos de inversión de mayor contribución al aumento en los niveles de productividad, tanto del capital como del trabajo, son proyectos de mediano y largo plazo. El estudio de Mckinnon (1973) muestra que el crecimiento y el desarrollo económico están estrechamente ligados a mercados financieros eficaces y flexibles. Eficaces en el sentido de que puedan proveer servicios de intermediación crecientes en volumen y decrecientes en costos para los usuarios de dichos servicios.

Estudios sobre la eficacia de los intermediarios financieros utilizan el MI (la diferencia entre la tasa cobrada sobre los préstamos y la tasa pagada sobre los depósitos) para explicar niveles de eficiencia en el sector (Barajas et al. 1999; Randall 1998; Catão 1998 y Mlachila y Chirwa 2002). En los indicados estudios se muestra como los intermediarios financieros latinoamericanos mantienen MI por encima de los prevalecientes en economías desarrolladas.

Brock y Rojas-Suárez (2000) determinaron que el MI en Latinoamérica se mantenía a niveles muy superiores de los 
que registran las economías desarrolladas, en la década de los 90. Los casos de Argentina, Perú, Colombia, Méjico, y Bolivia, aun después de introducidas importantes reformas en sus sistemas financieros, mostraban MI comprendido entre valores mínimos en la vecindad del $6 \%$ hasta valores máximos de 20\%, aproximadamente. Países industrializados; tales como: Suiza, Estados Unidos, Francia, Gran Bretaña, España e Italia, registran variaciones del MI, en los noventa, limitadas por un máximo de $5 \%$ y un mínimo próximo al $2 \%$. Los datos presentados implican diferencias promedios de $10 \%$ entre los países latinoamericanos y las economías desarrolladas.

Randall (1998) en su estudio sobre el MI en la banca comercial de las pequeñas islas del Caribe ( St. Kitts, Nevis, Antigua, Barbuda, Dominica, Grenada, St, Lucia, St. Vincent, las Granadinas, Montserrat y Anguilla) determinó diferencias, con los márgenes prevalecientes en Estados Unidos y Gran Bretaña de magnitudes entre $5 \%$ y $6 \%$.

La situación en el mercado financiero local no es muy diferente, de acuerdo con cifras publicadas por el Banco Central, en su portal de Internet, la media correspondiente a la tasa preferencial de préstamos para el periodo 1996-2002 es $21.5 \%$ y el promedio correspondiente a los certificados financieros es de $15.8 \%$. Él MI implícito asciende al 6\%, aproximadamente. El hecho de que los intermediarios financieros ofrecen préstamos a diferentes tasas y captan fondos por medio de diferentes instrumentos de captación, plantea ciertos retos metodológicos para el cálculo del MI. Estos aspectos se discuten mas adelante.

En este segundo artículo se amplía el análisis de los efectos de los costos de intermediación y el poder de mercado de los bancos comerciales sobre los márgenes de intermediación. La segunda parte de este trabajo resume datos de la ban- 
ca comercial como son: márgenes de intermediación (MI), índices de concentración de mercado y participación en el total de depósitos de los diferentes bancos. La tercera parte resume el marco conceptual sobre el cual se apoyan las estimaciones de los efectos que tiene el grado de poder de mercado de los intermediarios y su estructura de costos, en el MI. La sección siguiente presenta los resultados empíricos y sus implicaciones. La última sección incluye las conclusiones y recomendaciones de política económica.

\section{MÁRGENES DE INTERMEDIACIÓN: Repasan- do las definiciones. Cifras de la Banca Comercial}

Las definiciones del MI utilizadas en ambos artículos son las siguientes:

$$
\begin{aligned}
& \text { MI1 }=(\mathbf{I a} / \mathbf{A i})-(\mathrm{Ip} / \mathrm{Pi}) \\
& \text { MI2 }=(\mathbf{I a} / \mathbf{A i})-(\mathbf{I p} / \mathbf{A i})
\end{aligned}
$$

Donde: Ia es el total de ingresos por intereses; Ai es el total de activos que generan ingresos por intereses (cartera de crédito e inversiones). Ip representa todos los pagos por intereses. Pi es el total de pasivos que implican pagos por intereses (depósitos provenientes del público y préstamos obtenidos para operaciones de intermediación). MI2 es el margen bruto sobre los activos que generan ingresos por intereses. Otras definiciones pueden ser encontradas en la literatura sobre el tema. Brock y Rojas-Suárez (2000) resumen diferentes definiciones para el MI.

El Cuadro 1 muestra MI2 y MI1. Para la Banca Comercial estimados utilizando cifras consolidadas, para el período 1982-1986 y 1995-2001. Las cifras obtenidas muestran márgenes de intermediación por encima de los observados en economías desarrolladas. El promedio de MI2, para la banca 
comercial, aumentó de un $5.08 \%$ a un $9.49 \%$, en el período 1995-2001. La tasa activa promedio (definida en la ecuación 2) aumentó en un $10 \%$, y la pasiva promedio en un $4.41 \%$. Una situación parecida se registra en los cambios de las tasas correspondientes a las Asociaciones. Los aumentos en las tasas activas por encima de las pasivas, que caracteriza el período 1995-2001, parecen indicar la existencia de un mayor poder de mercado en la banca.

Márgenes de intermediación elevados han sido asociados con elementos de poder de mercado por parte de los intermediarios. Elevada participación en los totales de depósitos provenientes del público, de pocos intermediarios; es decir, el grado de concentración que se observa en la industria, en un factor que inciden sobre el MI (Mlachila y Chirwa 2002; Catão 1998). La realidad subyacente en la concentración de mercado es la que algunos intermediarios financieros puedan efectivamente influir sobre las tasas de interés (activas y pasivas); por ende, sobre el MI.

Una forma de medir esta concentración de mercado es calculando el índice de Herfindahl-Hirshman (HH). El índice se estima sumando las participaciones relativas del total de depósitos de cada banco en el total correspondiente al universo de los depósitos, elevadas al cuadrado, para un año determinado. El límite superior de este índice es de 10,000. Este valor correspondería al evento de que existiese un solo intermediario, con una participación en los depósitos del 100\%. Si el mercado es muy competitivo y existen muchos intermediaros con participaciones pequeñas, el valor del índice se aproxima a cero (Gelos y Roldós 2002).

El trabajo de Catão (1998) y el arriba citado, detallan las estimaciones $\mathrm{HH}$ para los casos de Argentina y otros países. El artículo de Gelos y Roldos (2002) discute los efectos de 
la consolidación bancaria en los llamados mercados emergentes sobre la estructura del mercado financiero. En adición al índice $\mathrm{HH}$, la participación en el total de los depósitos de los tres intermediarios principales, permite apreciar también la magnitud de la concentración en el mercado.

El Cuadro 2 presenta un comparativo de los índices de concentración, número de bancos y el porcentaje de los depósitos en los tres bancos principales. La comparación se hace para los años 1994 y 2000. Los índices de concentración para Brasil y Méjico se estimaron en el orden de 1278.6 y 1360.5 , respectivamente. La participación de los tres bancos principales, en el total de depósitos era de $55.2 \%$ para Brasil y $56.3 \%$ para Méjico.

En el caso de la República Dominicana, para el año 2000 los bancos comerciales presentan índices de concentración ascendentes a 1524.17. La participación de los tres principales bancos, en el total de los depósitos, promedió $58.96 \%$. Para economías más pequeñas que Brasil y Méjico, los autores antes citados estimaron índices de concentración en Malasia (1005.1), Filipinas (789.9) y Tailandia (854.4), también para el año 2000. Se puede observar que nuestros índices superan los presentados anteriormente.

El promedio del índice de concentración para los países latinoamericanos, en el 1994, fue de 958.56; mientras que, el índice correspondiente a la República Dominicana es de 1496.7. Los tres principales bancos, en los países latinos indicados, sólo administran un promedio equivalente a un 44.14\% del total de los depósitos, en el 1994. Para el 2000, Malasia, Tailandia y Korea, con un número equivalente de bancos comerciales al de la República Dominicana, registraron índices de concentración menores. En la distribución de los depósitos en la banca nacional, se observa que los tres 
bancos principales registran mas del $55 \%$ de los depósitos provenientes del público. Los once bancos restantes se reparten el $45 \%$ del mercado (Veloz, 2001).

En el Cuadro 3 se presentan los porcentajes de la participación de mercado, de los tres bancos principales, el índice de concentración y el porcentaje de margen de intermediación. En la porción inferior del indicado Cuadro, también se incluye la matriz de coeficientes de correlación entre las variables antes mencionadas. Aunque la simple observación de los datos en el Cuadro 3 no permite ver la dirección del grado de asociación entre las variables. La matriz de correlación si permite apreciar que los cambios en el índice de concentración $(\mathrm{HH})$ y los aumentos en el margen de intermediación (MI2) están positivamente correlacionados (0.50). De igual forma, se observa una mayor correlación, positiva, entre la participación de mercado (PM) y el margen de intermediación (MI2) equivalente a 0.57 .

\section{MARCO CONCEPTUAL}

$\mathrm{El}$ artículo anteriormente publicado discute, desde el punto de vista de organización industrial (Freixas \& Rochet 1999), la influencia de la estructura del mercado sobre los márgenes de intermediación. Se determinó como las magnitudes de las elasticidades influyen en el grado de poder de mercado de los intermediarios. La Gráfica 1 muestra como los cambios en los coeficientes de elasticidad de la oferta y demanda de fondos prestables determina la magnitud del MI. La gráfica en la izquierda muestra las curvas de oferta y demanda de fondos prestables más inelásticas que la gráfica sobre la derecha. La diferencia entre ia - ip (tasa activa menos tasa pasiva) es mayor en la primera que en la segunda. Para fines de la gráfica se asume que los costos margina- 
les no financieros son constantes. Dado la existencia de poder de mercado, el nivel óptimo de colocación y captación de fondos se obtiene igualando costo marginal $(\mathrm{cm})$ e ingreso marginal (D').

El análisis gráfico nos permite plantear otras preguntas de índole conceptual, independientemente del conocimiento exacto que se tenga sobre las magnitudes de las elasticidades. En un contexto temporal, un intermediario con poder de mercado puede: 1) aumentar el volumen de sus operaciones con aumentos simultáneos de su margen de intermediación. 2) puede también aumentar sus operaciones sin que se altere el margen de intermediación ó 3) ver disminuir sus márgenes con el aumento de las operaciones. El horizonte de tiempo a lo largo del cual un intermediario logre verse en las situaciones 1 y 2 , implica diferentes grados de poder de mercado.

Un banco comercial trata de expandir el espacio de tiempo entre los ajustes en las tasas pagadas sobre los depósitos y las cobradas sobre los préstamos. La regla implica que las tasas pasivas han de descender más rápido que las activas. Ante aumentos de tasas en el mercado, los movimientos de las tasas activas han de anteceder al aumento de las pasivas. En otras palabras, los aumentos en ingresos deben preceder, con suficiente margen temporal, a los aumentos en costos. Las implicaciones que esta lógica encierra son que los movimientos temporales del MI y el volumen de operaciones $\mathbf{F}$ se influyen, mutuamente. De esta forma añadimos otra aproximación para medir el poder de mercado. El procedimiento a seguir implica estimaciones, de las variables antes indicadas, con vectores auto regresivos que se describe en la siguiente sección.

Para caracterizar la naturaleza de la estructura del mercado, se utiliza la metodología desarrollada por Panzar y Ross 
(1987). La metodología indicada se basa en el equilibrio competitivo de largo plazo, el cual se caracteriza por la noexistencia de beneficios extraordinarios (Pindyck y Rubinfeld 2001). En otros términos, el monto total de los ingresos totales (IT) que resulta de las operaciones es exactamente suficiente para pagar el costo de oportunidad (CT) de todos los factores productivos empleados. Tanto la naturaleza del equilibrio competitivo como la existencia de funciones producción homogénea de grado uno soportan este resultado.

\section{MÉTODO DE ESTIMACIÓN}

Con la finalidad de estimar el efecto del poder mercado y los costos no financieros sobre el MI se utiliza la siguiente ecuación:

$$
\mathrm{MI}=\beta \mathbf{o}+\beta \mathbf{1}(\mathbf{p m})+\beta 2 \mathrm{w}+\beta 3 \mathrm{r}+\varepsilon \text { (3) }
$$

Donde (pm) es la participación en el total de los depósitos de los tres principales bancos. w es el gasto en personal y directorio sobre el total de activos y $\mathbf{r}$ representa gastos de capital medidos como el porcentaje de gastos generales y administrativos en el total de activos fijos. $\varepsilon$ es el error de estimación. La ecuación anterior se estima con variables dependientes MI2 y MI1.

La siguiente ecuación trata de capturar los cambios porcentuales en los ingresos por intereses ante cambios porcentuales en el costo de fondos, costos de mano de obra y costos de capital (Panzar y Ross 1987).

$$
\operatorname{Ln}(\mathrm{Ia} / \mathrm{Ai})=\lambda 0+\lambda 1 \operatorname{Ln}(\mathrm{Ip} / \mathrm{Ai})+\lambda 2 \operatorname{Ln}(\mathrm{w})+\lambda 3 \operatorname{Ln}(\mathrm{r})+\varepsilon(4)
$$

Si la suma de las elasticidades ingreso (Ia/Ai) con respecto al costo de fondos (Ip/Ai), costos de mano de obra (w) y costos de capital (r) es igual a la unidad, el mercado se 238 
puede caracterizar como competitivo. Los resultados posibles de las sumas de las elasticidades podemos resumirlas de la siguiente forma:

$$
\begin{array}{ll}
\lambda 1+\lambda 2+\lambda 3=1 & \text { (Mercado competitivo). } \\
\lambda 1+\lambda 2+\lambda 3<0 & \text { (Monopólico) }
\end{array}
$$

$0<\lambda 1+\lambda 2+\lambda 3<1$ (Competencia monopolística)

Los datos para las ecuaciones (3) y (4) se obtienen de conformar un pool de las estadísticas de los tres mayores bancos comerciales: Banco Popular, Banco de Reservas y Banco Intercontinental. Las observaciones temporales correspondientes son trimestrales, iniciando en diciembre del 1995 y terminan en septiembre del 2000. Para mayores detalles sobre el pooling referirse a Pindyck y Rubinfeld (1981) y Eviews User's Guide (1994 -1997). La razón principal para la aplicación del pooling es la no-existencia de series de tiempo lo suficientemente largas y consistentes.

Pruebas adicionales sobre el poder de mercado en la banca comercial serán realizadas, con datos mensuales, mediante estimación VAR (Gujarati 2000). Las estimaciones con vectores auto-regresivos (VAR) toman la siguiente representación:

$$
\begin{aligned}
& \text { 'j j } \\
& \mathbf{M I}=\delta+\sum \beta \mathbf{h ~ F t - h}+\sum \tau \mathbf{h} \mathbf{M I} \mathbf{t}-\mathbf{h}+E \mathbf{t} \\
& \text { ' } h=1 \quad ' h=1 \\
& \text { j j } \\
& \mathbf{F}=\delta^{\prime}+\sum \mu \mathbf{h} \mathbf{F t}-\mathbf{h}+\sum \theta \mathbf{h M I} \mathbf{t}-\mathbf{h}+\xi \mathbf{t} \\
& \text { ' } h=1 \quad ' h=1
\end{aligned}
$$

La estimación VAR a ser efectuada no busca establecer simultaneidad entre las variaciones en los fondos prestables 
(F) y los márgenes de intermediación (MI). La pregunta a discutir es que dado cierto poder mercado hasta donde los valores de los márgenes actuales pueden mantenerse con previos aumentos en las operaciones con fondos prestables, o no ver disminuidas estas operaciones, ante aumentos previos en los márgenes. La longitud del tiempo sobre el cual los coeficientes, en conjunto, de las ecuaciones (5) y (6) mantengan niveles aceptables de confiabilidad permite inferir sobre el grado de poder de mercado. En otras palabras, un banco que puede por tres meses ver aumentar sus márgenes y operaciones, simultáneamente, posee mayor poder de mercado que otro banco cuyas operaciones y márgenes sólo aumenta por un mes. F en la estimación VAR es definido como el total de activos.

\section{RESULTADOS EMPÍRICOS}

El Cuadro 4 resume los resultados de las estimaciones de las ecuaciones (3) y (4). Los valores de los coeficientes se resaltan en negritas y los números debajo de estos corresponden a los valores t y p. Aquellos coeficientes con valores $\mathrm{p}$ por debajo de 0.05 son estadísticamente significativos.

Los cambios en la participación de mercado están positivamente correlacionados con los aumentos en los márgenes. Sin embargo, los coeficientes estimados no resultaron estadísticamente significativos. La existencia de multicolinealidad entre w y $r$ influye en este resultado. De la misma forma que luce influir sobre el signo del coeficiente correspondiente a w. La influencia de los aumentos en los costos de capital resultan positivamente asociados con los aumentos en los márgenes. El coeficiente estimado es estadísticamente significativo. 
En cuanto a la naturaleza del poder de mercado, la suma de las elasticidades del ingreso por intereses con relación al costo de fondos, al costo de mano de obra y al costo de capital es menor que la unidad y mayor que cero (véase las condiciones establecidas en la ecuación 4 , y la ecuación 3 del Cuadro 4). Esto permite caracterizar la naturaleza del mercado como competencia monopolística, y por ende los intermediaros pueden influir sobre los márgenes de intermediación.

De nuevo el signo de Ln w en la ecuación 3 (Cuadro 4) resulta negativo y estadísticamente significativo. Las elasticidades estimadas para los cambios en los ingresos por intereses (Ln Ia) ante cambios en los costos de fondos ( $\mathrm{Ln} \mathrm{Ip)}$ y cambios en los costos de capital son positivas, y estadísticamente significativas. El signo negativo del coeficiente de elasticidad de los ingresos, con relación a los costos de mano de obra, luce indicar que los bancos han sido capaces de traspasar, vía aumentos de las tasas activas, los aumentos en las tasas pasivas y en costos de capital; pero los aumentos en los costos laborales han implicado presiones a la baja, en los márgenes de intermediación.

Al igual que en los trabajos de Randall (1998), Mlachila \& Chirwa (2002) y Brock \& Rojas-Suárez (2000) las estimaciones resultan sensibles a la definición que se adopte de los márgenes de intermediación. En específico podemos ver un cambio en el nivel de significación del coeficiente que mide el impacto del aumento de los costos de mano de obra sobre los márgenes de intermediación. Las ecuaciones 1 y 2 muestran el cambio en el coeficiente antes indicado cuando se sustituye MI1 por MI2. El coeficiente asociado con los costos de capital ( $r$ ) resulta significativo en ambas ecuaciones.

En lo que concierne a la participación de mercado, para el caso de la banca comercial adoptamos el porcentaje de parti- 
cipación en el total de los depósitos del sistema de los tres bancos incluidos en el pool. Aunque el signo de esta variable es positivo en ambas ecuaciones, creemos que la poca variabilidad que ésta presenta en los datos trimestrales, le hacen perder significación estadística. Sin embargo los resultados obtenidos en la ecuación 3, donde incluyese las elasticidades de los ingresos por intereses con relación a los cambios en las tasas pasivas, costos de personal y de capital, si se señalan la existencia de poder de mercado.

Es apreciable ver que en el período analizado los bancos han podido traspasar a las tasas activas la mitad de los aumentos en las tasas pasivas. El coeficiente de elasticidad en cuestión es 0.5320 , y significativo al $5 \%$. Los problemas de multicolinealidad entre $\mathrm{w}$ y $\mathrm{r}$ distorsionan el valor del signo esperado del coeficiente para w. Es posible también que los continuos aumentos en el costo de personal en la banca pudiese haber tendido reducir márgenes.

Ante esta situación es que sugerimos la estimación VAR descrita en las ecuaciones (5) y (6) como una forma de producir mas información sobre el poder de mercado de los intermediarios financieros. El Cuadro 5 resume las estimaciones realizadas para el Banco Popular, Baninter y BHD. Los retrasos en las variables márgenes de intermediación y cambios en el volumen de operación son tres. Las cifras seleccionadas para cada banco corresponden a observaciones mensuales durante el período 2001-2002. Los coeficientes estimados se presentan en letras negritas. Los números inmediatamente debajo de los coeficientes son las estadísticas t.

El caso extremo donde el volumen de operaciones y los márgenes aumenten simultáneamente no se observa para ningunos de los tres bancos. En el caso del BHD, los aumentos en los márgenes han podido registrarse, por dos me- 
ses consecutivos, independientemente de las variaciones en el volumen de las operaciones. En lo concerniente al Banco Popular, el coeficiente negativo en la columna 4 de la primera ecuación no nos permite concluir de forma similar al BHD. Los efectos de los cambios precedentes en los volúmenes de operaciones sobre los valores actuales de la misma variable son negativos, y estadísticamente significativos. La información que las estimaciones VAR proveen señala la posibilidad de cambios significativos en los coeficientes de elasticidad de las curvas de demanda y oferta de fondos prestables individuales, en el corto plazo.

\section{CONCLUSIONES}

Los resultados obtenidos en las estimaciones empíricas no descartan la existencia de poder de mercado en la banca comercial. El grado de concentración observado es superior en las AAyP que en los bancos comerciales. Los índices de concentración estimados sobrepasan a los correspondientes al sistema bancario de otros países de Latinoamérica y de economías más pequeñas como las islas del Caribe Inglés.

Es visualizable que un mayor grado de competitividad resultaría en una mayor eficiencia en la intermediación financiera; y por tanto, una posible reducción en los márgenes de intermediación. En la realidad, la banca comercial ha visto pasar procesos de ventas y adquisiciones de instituciones y la entrada de nuevos bancos. Estos eventos han hecho variar los índices de concentración observados. Sin embargo, el tamaño de los nuevos participantes en el mercado bancario es tal que no implican un reto para los bancos principales y su poder de mercado. La red de sucursales que los nuevos participantes traen al mercado resulta de imperceptible influencia sobre el grado de competencia. 
Desde el punto de vista metodológico, la utilización del índice $\mathrm{HH}$ y la participación en el mercado como variables próximas para medir poder de mercado no lucen superiores a la estimación, en forma simultánea o independiente, de las elasticidades de la oferta y demanda de fondos prestables (Randall 1998). De nuevo el reto radica en determinar la tasa de interés o las tasas relevantes para explicar la demanda de fondos prestables. Estimar la variabilidad de los coeficientes de elasticidad para diferentes plazos, e individualmente para cada banco, será también necesario.

En cuanto a la oferta de fondos prestables, la tasa sobre los certificados de depósitos parece la tasa a ser utilizada para calcular el coeficiente de elasticidad correspondiente. En este caso sería necesario ponderar la tasa de cada banco por el porcentaje de participación en los depósitos de cada uno. Otra manera de identificar la función de oferta de fondos puede ser estimando tasas medias mensuales por banco, como variable independiente. Como variable dependiente los cambios en los saldos en el total de depósitos mensuales. Esto es tema de una futura investigación.

Bajo la situación de que los coeficientes estimados para medir la influencia de las variables de concentración de mercado, sobre los márgenes de intermediación, resultaron no significativas, estadísticamente, la metodología desarrollada por Panzar y Ross (1987) para someter a prueba la hipótesis sobre existencia de competencia monopolística resultó ser apropiada. Las estadísticas presentadas en la sección 2 de este trabajo mostraron índices de concentración y márgenes de intermediación, en nuestro sistema financiero, con valores que sobrepasan los correspondientes a países donde se ha determinado relaciones estadísticamente significativas entre los niveles de concentración y los márgenes de intermediación. 
Es posible que los elevados márgenes de intermediación observados en el período bajo análisis estén asociados con la idea de maximizar el valor de la licencia o la franquicia financiera que se opera añadiendo elementos de renta en las operaciones. Esto es plausible en un ambiente donde las adquisiciones y fusiones han estado tocando la puerta de los bancos. Otro aspecto de importancia indicado por los resultados empíricos, es la influencia que los aumentos en los costos laborales han tenido sobre los márgenes de intermediación. Los resultados parecen indicar que el ejercicio de poder de mercado no haya permitido traspasar los aumentos en costos laborales a las tasas activas. Otra interrogante que surge es si la remuneración de laboral en el sector de la banca refleja el costo de oportunidad de ésta.

Si revisamos rápidamente la evolución de los mercados financieros en economías desarrolladas podemos ver que fue la aparición y desarrollo de otras formas de intermediación financiera lo que contribuyó a la reducción de los márgenes de intermediación. En específico ha sido el desarrollo de los mercados de capitales (bolsas de valores) lo que ha permitido que la oferta y demanda de fondos prestables se efectúe con costos de transacción mas reducidos. Inversionistas y ahorrantes intercambian, prácticamente, en forma directa y contractualmente simple, fondos. Particularmente opino que es auspiciando un rápido desarrollo de los mercados de capitales, que desde el punto de vista micro, se logrará tener mayores transacciones financieras a menor costo.

\section{BIBLIOGRAFÍA}

Barajas, Adolfo; Steiner, Roberto \& Salazar, Natalia. 1999; "Interest Spread in Baking in Colombia, 19974-1996". IMF Staff Papers. Vol. 46. No. 2. June. 
Brock, Phillip \& Rojas-Suarez, Liliana, 2000. Editors; "Interest Rate Spreads in Latin America: Facts, Theories and Policy Recommendations". Published in WHY SO HIGH? Interamerican Development Bank.

Brock, Phillip \& Rojas-Suarez, Liliana, 2000. "Understanding the Behavior of Bank Spreads in Latin America". Journal of Development Economics. Vol 63, pp. 113-134.

Catão, Luis; 1998; "Intermediation Spreads in a Dual Currency Economy Argentina in the 90's"; IMF Working Paper No. 98/90. May.

Eviews User's Guide. 1994-1997, Quantitative Micro Software.

Freixas, Javier \& Rochet Jean - Charles. 1999. MICROECONOMICS OF BANKING. MIT Press. Cambridge, Mass.

Gelos, R. Gaston \& Roldós, Jorge 2002. "Consolidation and Market Structure in Emerging Market Banking Systems". IMF Working Paper WP/02/186. November.

Gujarati, Damodar N. 2000. ECONOMETRIA. Tercera Edición. McGraw Hill

McKinnon, R.I., 1973. MONEY and CAPITAL in ECONOMIC DEVELOPMENT. The Brookings Institution, Washington.

Mlachila, Monfort \& Chirwa, Ephraim W. 2002. "Financial Reforms and Interest Rate Spread in the Commercial Banking System in Malawi"; IMF Working Paper WP/02/6. January

Panzar, John C. \& Ross, James N. 1987, "Testing for 'Monopoly' Equilibrium", The Journal of Industrial Economics, Vol. 35, No. 4, pp-443-456.

Pindyck, Robert S. and Rubinfeld, 1981. ECONOMETRICS MODELS \& ECONOMIC FORECASTS. Second Edition. McGraw Hill.

Pindyck, Robert S. and Rubinfeld, Daniel. 2001. MICROECONOMIA. Prentice-Hall.

Randall, Ruby. 1998. "Interest Rate Spreads in The Eastern Caribbean". IMF Working Paper WP/98/59. April.

Veloz, Alberto. 2001. "Los coeficientes de Elasticidad Costo y las Inexistentes Economías de Escala en La Banca Dominicana". Ciencia y Sociedad, 25(4): 457-72, oct.-dic. 


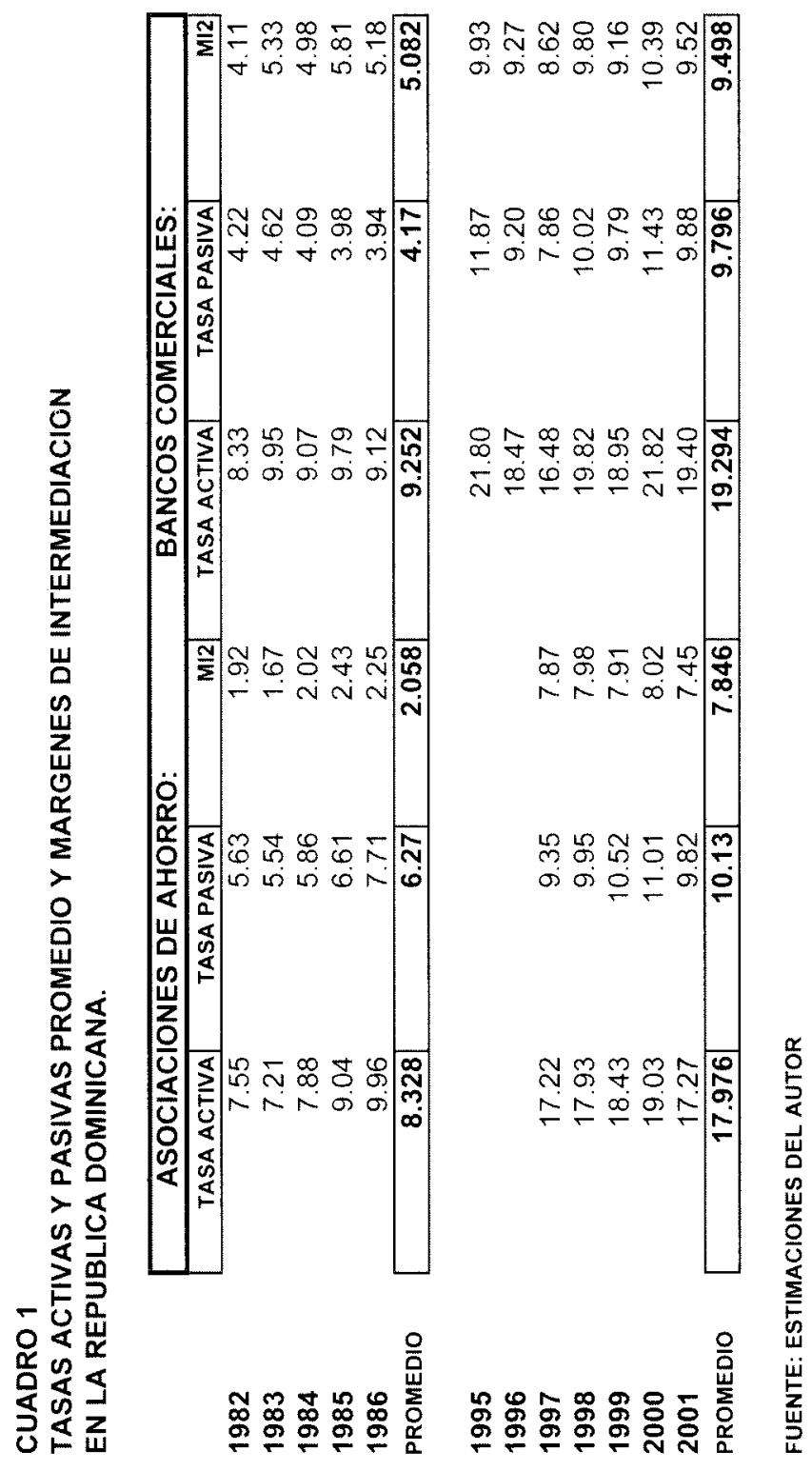




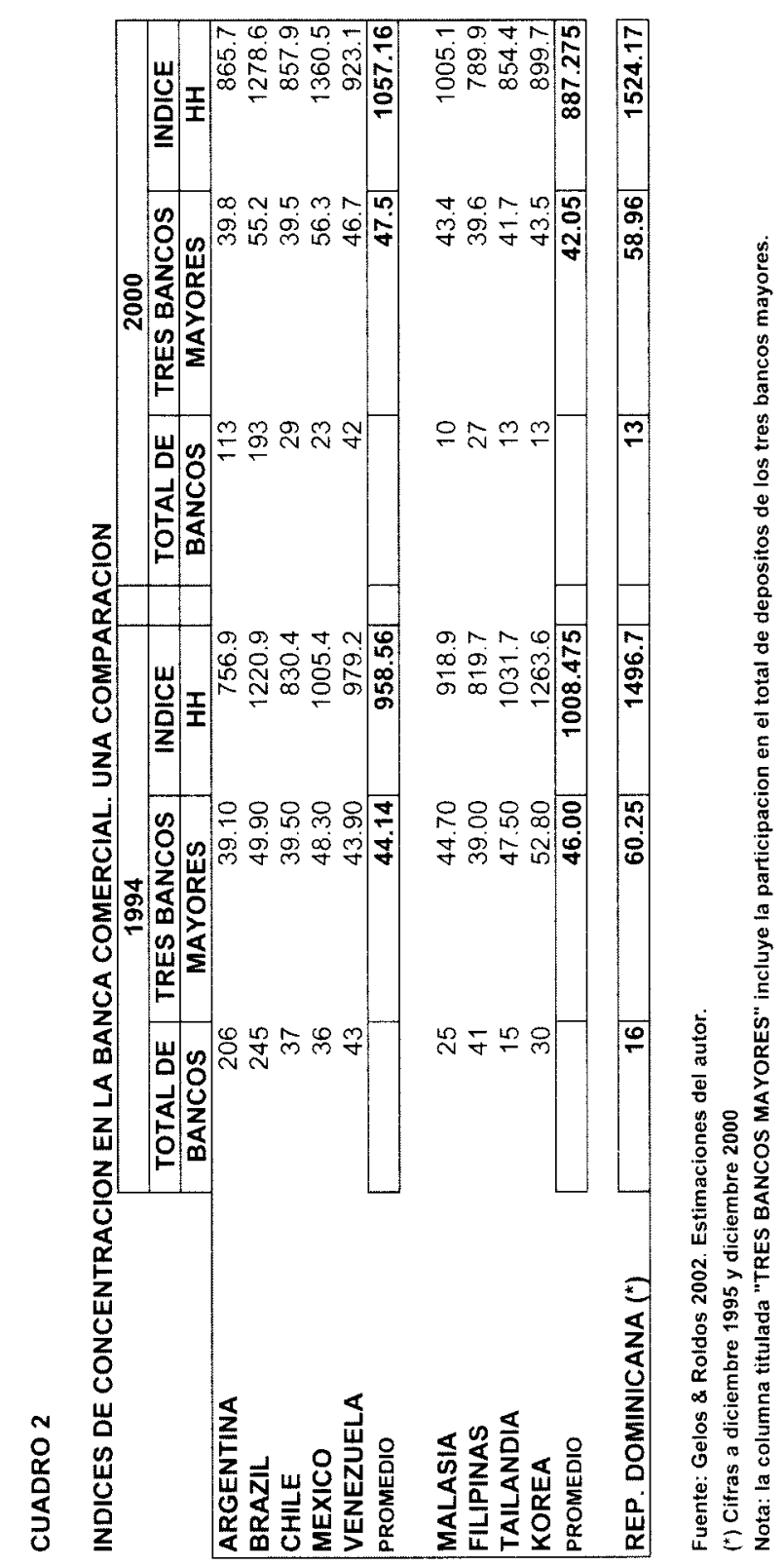




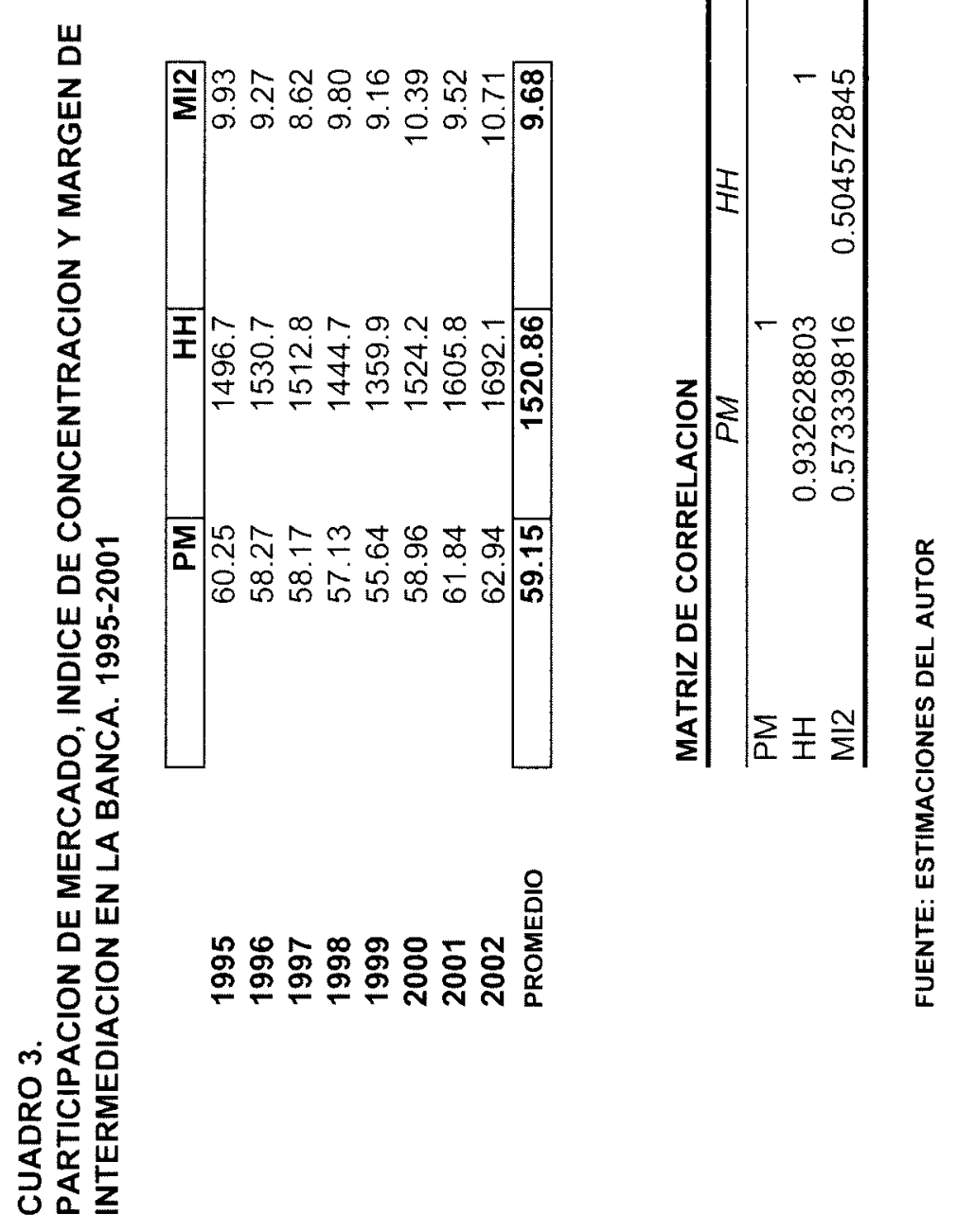




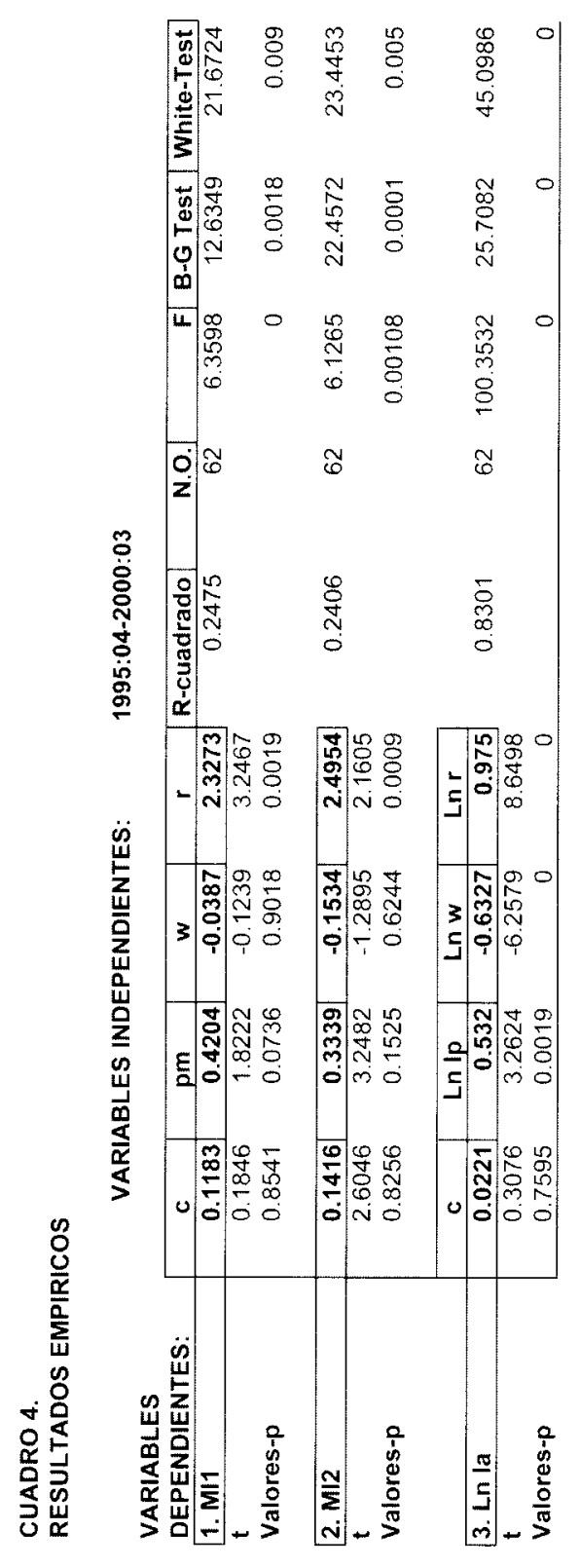




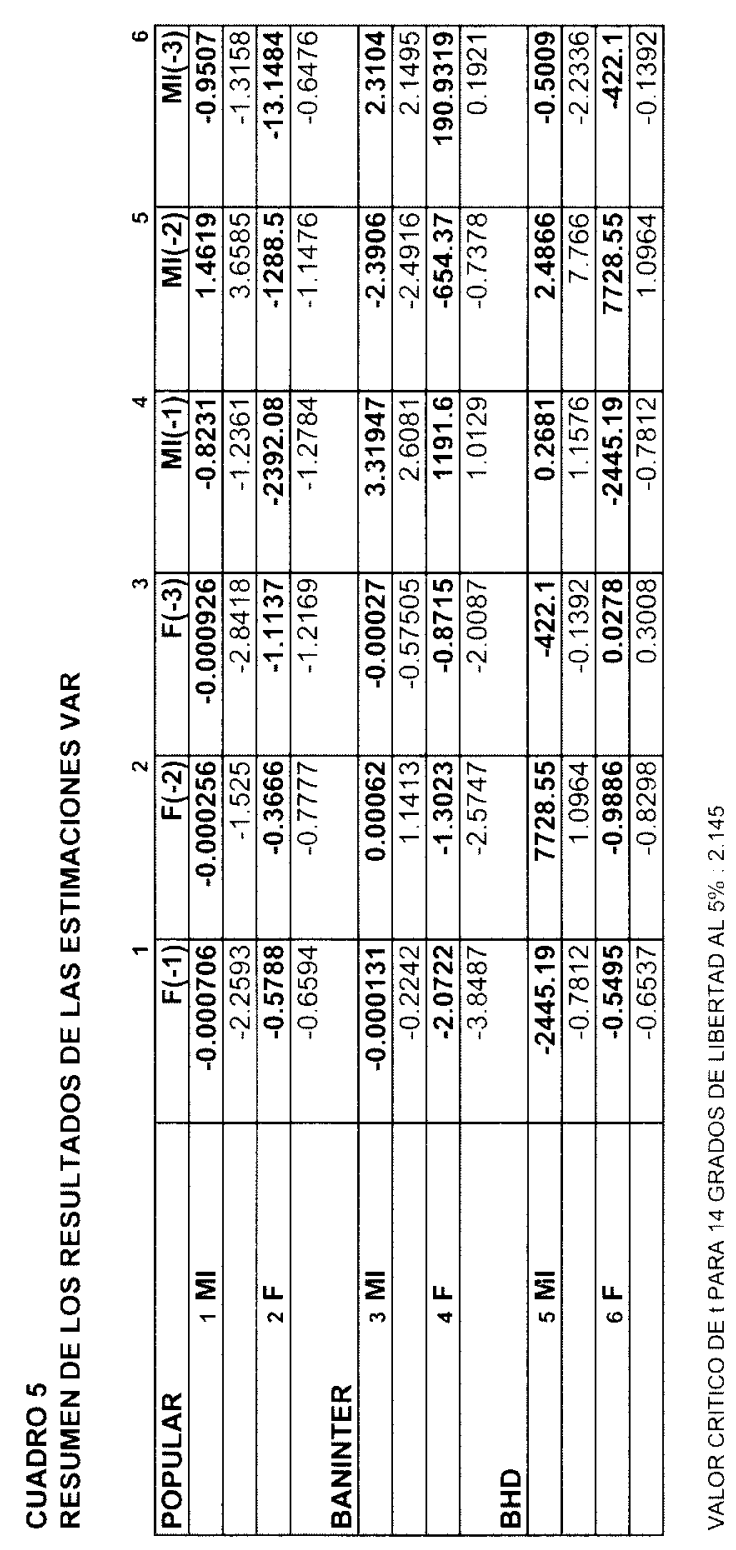




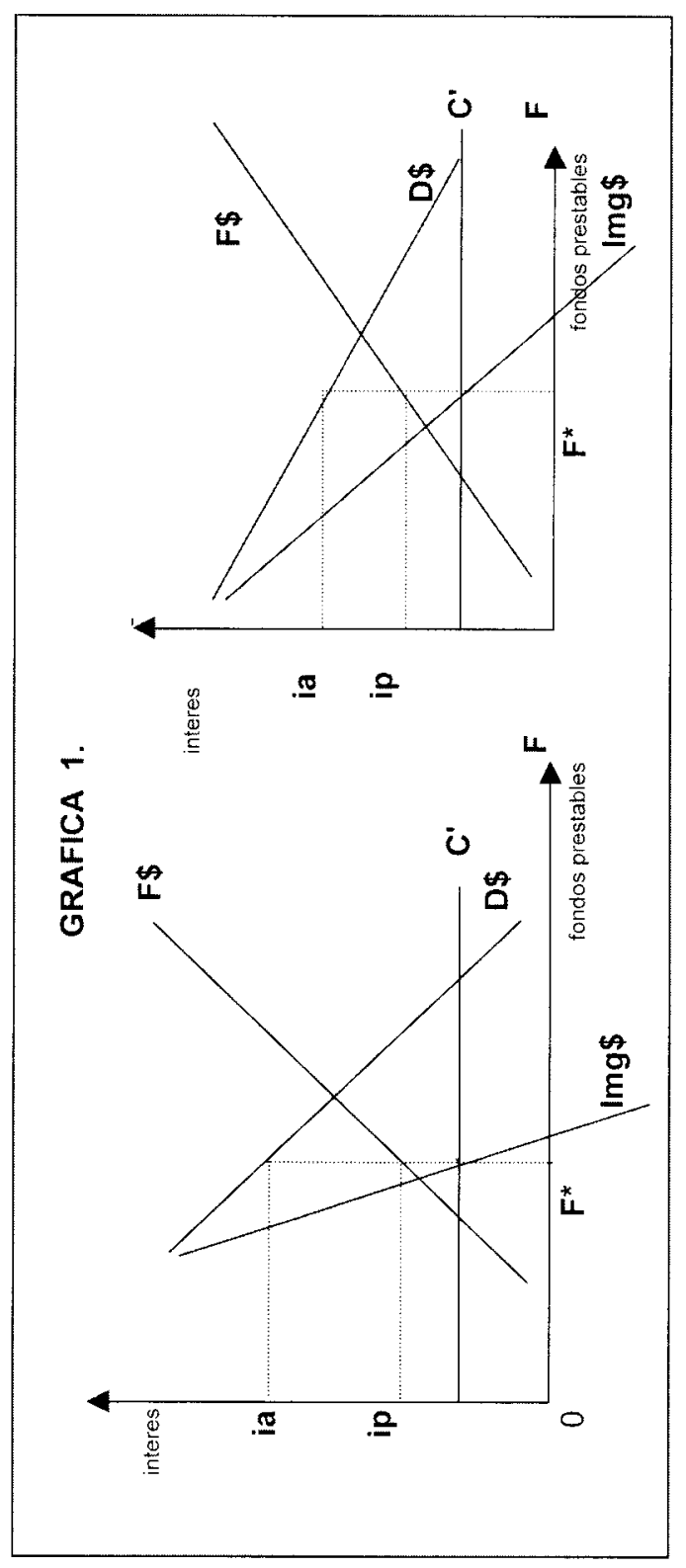

